\title{
Visualizing the Spatial Distribution of Ripples in Graphene with Low-Energy Electron Diffractive Imaging
}

\author{
I.-S. Hwang ${ }^{1,2}$, W.-H. Hsu ${ }^{1,2}$, W.-T. Chang ${ }^{1}$, C.-Y. Lin ${ }^{1}$, and T. Latychevskaia ${ }^{3}$ \\ 1. Institute of Physics, Academia Sinica, Nankang, Taipei, Taiwan. \\ 2. Department of Materials Science and Engineering, National Tsing Hua University, Hsinchu, Taiwan. \\ 3. Physics Department of the University of Zurich, Zürich, Switzerland
}

Graphene has received much attention owing to its outstanding electrical and mechanical properties. Ripples are an intrinsic feature of graphene sheets. Meyer et al. reported evidence of the intrinsic ripples in the form of broadened diffraction spots in reciprocal space [1]. However, real-space imaging of graphene rippling remains challenging. Such imaging may allow better understanding of origin and dynamics of the rippling. In this work, we demonstrate that graphene ripples can be visualized with an electron point projection microscopic/diffractive imaging instrument. Through numerical simulations, we showed that three-dimensional displacement of carbon atoms as small as $1 \AA$ can be detected with electrons of low-energy (50-500 eV).

Fig. 1 shows a schematic of our low-energy electron point projection microscopic/diffractive imaging instrument. It have an arrangement almost identical to a low-energy electron point projection microscope (PPM) [2]. During operation of a PPM, the sample is grounded and an electron emitter (a sharp tip) is negatively biased to extract electrons from the tip apex. The electron beam illuminates the sample (usually a thin object) and the transmission pattern is projected on a screen consisting of a microchannel plate (MCP). The magnification of the image is equal to the ratio of the source-screen distance $(D+d)$ to the source-object distance $(d)$, i.e. magnification $M=(D+d) / d$. The emitter is mounted on a three-axis piezo-driven nano-positioner with a traveling distance of $5 \mathrm{~mm}$ in each direction. The unique part of our setup in Fig. 1 is that the screen is mounted on a rail such that $D$ can be changed from $3 \mathrm{~mm}$ to $130 \mathrm{~mm}$. We can record high-magnification images when $D$ is large and high-angle diffraction patterns when $D$ is small. The emitter we use here is a $\operatorname{Ir} / \mathrm{W}(111)$ single-atom emitter $[3,4]$. This emitter has been demonstrated to provide high brightness and fully spatially coherent electron beams with Gaussian distributed intensity profiles and a full divergence angle of $2-6^{\circ}[3,4]$.

Fig. 2(a) shows a diffraction pattern we have recorded on a monolayer graphene. The center region is the zero-order spot formed by the electron wave transmitted through the suspending graphene region. It is considerably brighter than the six surrounding first-order spots, which are related to diffraction of a single domain of monolayer graphene. Fig. 2(b) shows the zero-order spot after adjusting the image contrast, which is the projection image or hologram of the sample. The dark distributions on the top and bottom edges are associated with aggregation of adsorbates on graphene, which are non-transparent for the electron beam. In the center region, clean graphene with only one or two darker or brighter spots corresponding to small individual adsorbates are evident. The dark distributions associated with adsorbate aggregates remain visible in the first-order diffraction spots [Fig. 2(a) and 2(c)]. One of the first-order diffraction patterns is shown with enhanced contrast in Fig. 2(c). Surprisingly, bright and dark stripes [two are indicated with white arrows in Fig, 2(c)] appear to connect between the dark adsorbate aggregates on the top and bottom edges. Notice that neither structures nor stripe patterns are seen in the corresponding zero-order spot [Fig. 2(b)], indicating that the stripes appear in the clean graphene region. In many of our experiments, we have observed stripes mainly formed between adsorbates. When the 
adsorbate patterns change over time, as seen in the zero-order spot, we also observed the corresponding changes in the distribution of the stripe patterns in the first-order spots.

We have performed numerical simulations of the diffraction patterns to characterize the observed stripe patterns [5]. Our simulations indicate that the contribution from the adsorbates and from threedimensional distribution of ripples in graphene can be separated by comparing the intensity in the zeroand the first-order diffraction spots. In addition, an out-of-plane ripple produces a similar intensity distribution among all six first-order diffraction spots, whereas an in-plane ripple produces various intensity distributions among the first-order diffraction spots. When imaging with low-energy electrons, the ripples of amplitude $1 \AA$ are sufficiently strong to cause noticeable intensity variations in the firstorder diffraction patterns. Thus, very weak ripples associated with strain caused by adsorbates on the graphene surface can be directly visualized and studied.

[1] JC Meyer et al., Nature 446 (2007) 60.

[2] HW Fink, W Stocker and H Schmid, Phys. Rev. Lett. 65 (1990) 1204.

[3] HS Kuo, IS Hwang, TY Fu, JY Wu, CC Chang and TT Tsong, Nano Lett. 4 (2004) 2379.

[4] CC Chang, HS Kuo, IS Hwang and TT Tsong, Nanotechnology 20 (2009) 115401.

[5] T Latychevskaia, WH Hsu, WT Chang, CY Lin and IS Hwang, Nature Communications 8 (2017) 14440.

[6] The authors acknowledge funding from Academia Sinica of R. O. C. (AS-99-TP-A02).

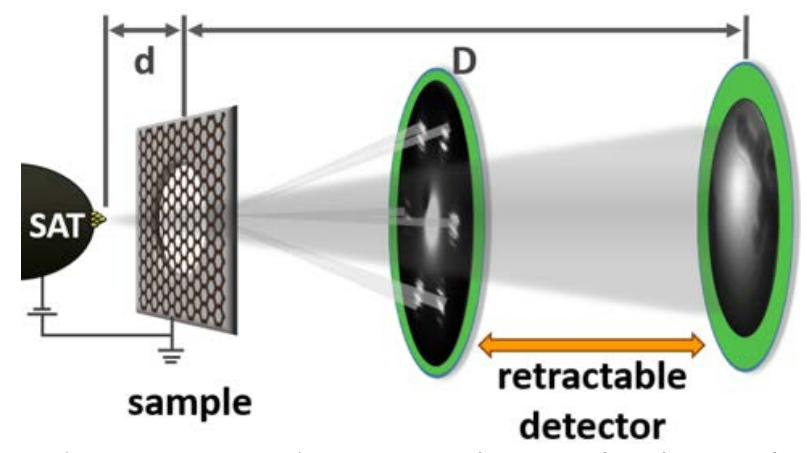

Figure 1. Schematic of a low-energy electron point projection microscopic/diffractive imaging instrument. It consists of a single-atom electron source, a suspended graphene sample, and a retractable detector.

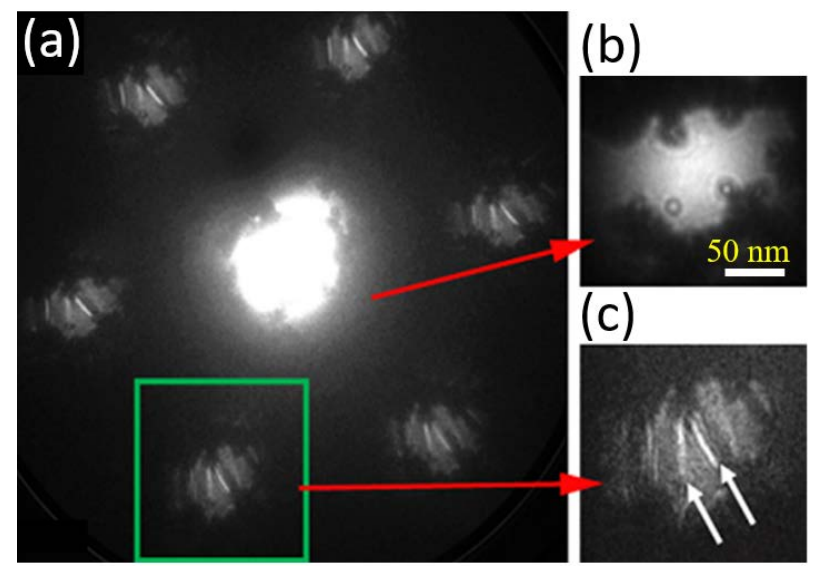

Figure 2. Diffraction pattern of a freestanding monolayer graphene recorded at $245 \mathrm{eV}$. 